\title{
Model Reference Adaptive Control Design for Nonlinear Plants
}

\author{
Wafa Ghozlane ${ }^{1}$, Jilani Knani ${ }^{2}$ \\ Research Laboratory in Automatic Control LA.R.A \\ National Engineers School of Tunis, ENIT, University of Tunis El Manar, BP 37, Le Belvédère, 1002, Tunis, Tunisia
}

\begin{abstract}
In this paper, the basic theory of the model reference adaptive control design and issues of particular relevance to control nonlinear dynamic plants with a relative degree greater than or equal to one with unknown parameters are detailed. The studied analysis was motivated through its application to a robot manipulator with six degrees of freedom. After linearization using the input-output feedback linearization and decoupling algorithm, the nonlinear Multi-input Multioutput system was transformed into six independent single-input single-output linear subsystems each one has a relative degree equal to two, the obtained results in different simulations shows that the augmented reference model adaptive controller has been successfully implemented.
\end{abstract}

Keywords-Model reference adaptive control; nonlinear dynamic plants; relative degree; unknown parameters; robot manipulator; input-output feedback linearization

\section{INTRODUCTION}

Nowadays, a great performance of industrial control systems are under adaptive control techniques [1], these include a high scale of tasks in aerospace, robotics, process control, ship steering, and automotive and biomedical plants.

Specially, for robotic control, a control designer can be faced with joint flexibilities, unknown manipulator dynamic parameters, nonlinear joint interactions, and dynamics changing due to unknown and varying loads. Traditional robotic control algorithms have depended on specific knowledge of the robotic parameters and dynamic equations [2]. When a designer has limited knowledge of these parameters and interactions, it can be advantageous to exploit adaptive control approaches to reduce the effects of these problems.

Generally, the model reference adaptive control system (MRAC) was initially developed to adjust the problems in which the performance specifications are given in terms of a reference model $[1,3,4]$. This model tells how the process output ideally should deal to the command signal. The structure of the control system is given in Fig. 1.

The controller may be thought of as composed of two loops, the inner loop is an regular feedback loop consisting of the plant and the controller, the outer loop error, which determines the difference between plant output and model output is small $[1,5]$. The MRAC was orginally introduced for advanced control. The crucial importance with MRAC is to analyse the adjustment mechanism so that a stable system which brings the error to zero, is obtained.

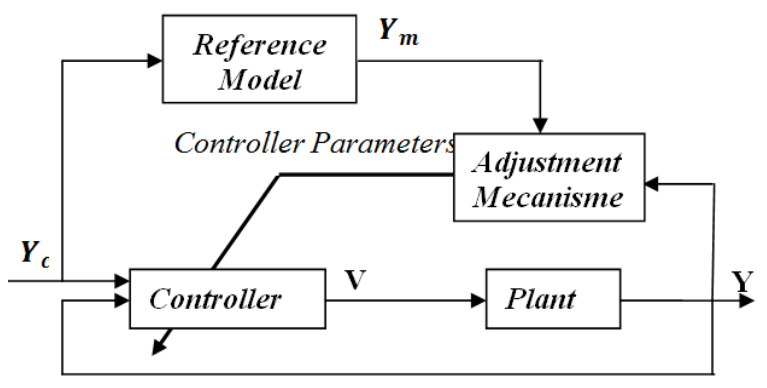

Fig. 1. A Model-Reference Adaptive Control System [1].

The organization of this paper is as follows: It is designed by five parts. In Section 2, the adaptive control statement is presented. In Section 3, the structure of the model reference adaptive control system with relative degree greater than or equal to one is explained. In Section 4, the control approach is applicated to a robot manipulator with six degrees of freedom and the simulation results are developed. Finally, the conclusion was detailed in Section 5.

\section{PRoblem Statement}

The problem under consideration [5], is the control of a single-input single-output (SISO) discrete time linear system which is elaborated by the input output $\{\mathrm{V}(\mathrm{k}), \mathrm{Y}(\mathrm{k})\}$ and can be formulated by the transfer function of the form:

$G_{p}\left(q^{-1}\right)=q^{-d} K_{p} \frac{B\left(q^{-1}\right)}{A\left(q^{-1}\right)}$

Where $A\left(q^{-1}\right)$ denotes a monic polynomial with degree $n, B\left(q^{-1}\right)$ represents a monic stable polynomial with degree $m<n$, the term $d=n-m$ is designed the relative degree of the system and $K_{p}$ is called a constant gain parameter.

A model reference is represented by the input output $\left\{Y_{c}(k), Y_{r}(k)\right\}$ and can be described by the transfer function.

$G_{m}\left(q^{-1}\right)=q^{-d} K_{m} \frac{B_{m}\left(q^{-1}\right)}{A_{m}\left(q^{-1}\right)}$ 
Where $A_{m}\left(q^{-1}\right)$ and $B_{m}\left(q^{-1}\right)$ represent respectively a monic stable polynomial with degrees $n$ and $m<n, K_{m}$ denotes a constant gain parameter.

Therefore as [6], the relative degree of the model is supposed to be greater than or equal to that of the system.

The purpose of the MRAC design is to determine a control law $\mathrm{V}(\mathrm{k})$, and an adaptation law, such that $[6,7]$ the resulting model following error $Y(k)-Y_{r}(k)$ asymptotically converges to zero, such that relation (3).

$$
\lim _{k \rightarrow+\infty}|e(k)|=\lim _{k \rightarrow+\infty}\left|Y(k)-Y_{r}(k)\right|=0
$$

\section{Structure Of the Model REFERENCE AdAPTiVE CONTROLLER}

The general structure of the model reference adaptive control system can be detailed as shown in Fig. 2 by the block diagram below.

Two identical block for generating auxiliary filter signal FSA1 and FSA2 both with dimension $n, W^{(1)}(k)$ and $W^{(2)}(k)$ with dimension $(\mathrm{n}-1) \mathrm{x} 1$ denote the vectors of state variables and $\mathrm{V}(\mathrm{k})$ and $\mathrm{Y}(\mathrm{k})$ represent respectively, the inputs of the designed controller as detailed in Fig. 2.

Consider the following state space representation of the SISO plant dynamics, together with two "signal filter generators" formed by a controllable pair $(\Lambda, B)$ are given as.

$$
\begin{aligned}
& \left\{\begin{array}{c}
\mathrm{X}_{\mathrm{p}}(\mathrm{k}+1)=\mathrm{A}_{\mathrm{p}} \mathrm{X}_{\mathrm{p}}(\mathrm{k})+\mathrm{B}_{\mathrm{p}} \mathrm{V}(\mathrm{k}) \\
\mathrm{Y}(\mathrm{k})=\mathrm{C}_{\mathrm{p}} \mathrm{X}_{\mathrm{p}}(\mathrm{k})
\end{array}\right. \\
& \left\{\begin{array}{c}
W^{(1)}(k+1)=\Lambda W^{(1)}(k)+B V(k) \\
V_{F}(k)=C^{T} W^{(1)}(k)
\end{array}\right. \\
& \left\{\begin{array}{c}
W^{(2)}(k+1)=\Lambda W^{(2)}(k)+B Y(k) \\
Y_{F}(k)=\bar{D}^{T} W^{(2)}(k)+d_{0} Y(k)
\end{array}\right.
\end{aligned}
$$

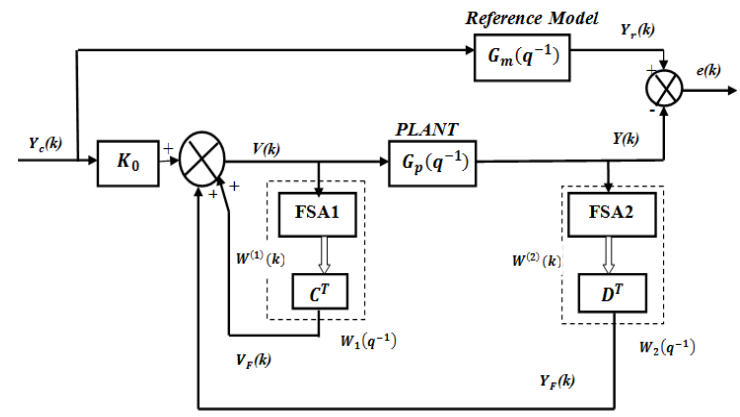

Fig. 2. Block Diagram of Model Reference Adaptive Control. where,

$$
\begin{gathered}
\Lambda=\left[\begin{array}{ccccc}
0 & 1 & 0 & \cdots & 0 \\
\vdots & \ddots & \ddots & \ddots & \vdots \\
0 & \cdots & 0 & 0 & 1 \\
-\mathrm{n}_{\mathrm{n}-1} & \cdots & \cdots & -\mathrm{n}_{2} & -\mathrm{n}_{1}
\end{array}\right], \quad B=\left[\begin{array}{c}
0 \\
0 \\
\vdots \\
0 \\
1
\end{array}\right], \\
C^{T}=\left[\begin{array}{llll}
c_{n-1} & \cdots & \cdots & c_{1}
\end{array}\right] \bar{D}^{T}=\left[\begin{array}{llll}
d_{n-1} & \cdots & \cdots & d_{1}
\end{array}\right] \\
C^{T}=\left[\begin{array}{lll}
c_{1} & \cdots & c_{n-1}
\end{array}\right] \text { and } D^{T}=\left[\begin{array}{lll}
d_{0} & \cdots & d_{n-1}
\end{array}\right] \\
\quad \text { represents the controller parameters. }
\end{gathered}
$$

According to the block diagram of the control system, the control law can be put in the following form:

$V(k)=K_{0} Y_{c}(k)+W_{1}\left(q^{-1}\right) V(k)+W_{2}\left(q^{-1}\right) Y(k)$

The expression (7) can be formulated as follows:

$V(k)=\theta^{T}(k) \varphi(k)$

Where

$$
\begin{aligned}
& \theta^{T}(k)=\left[\begin{array}{lll}
K_{C}(k) & C^{T}(k) & D^{T}(k)
\end{array}\right]=\left[\begin{array}{lllllll}
K_{C}(k) & c_{1}(k) & \cdots & c_{n-1}(k) & d_{0}(k) & \cdots & d_{n-1}(k)
\end{array}\right] \\
& \varphi^{T}(k)=\left[\begin{array}{lll}
Y_{c}(k) & V_{F}(k) & Y_{F}(k)
\end{array}\right]
\end{aligned}
$$

Finally, the calculation of the control law requires knowledge of the parameters of the system. However, in practice, these parameters are unknown and variable in time. So, the online estimation of the control parameters is therefore necessary $[6,7,8,9,10]$. In this case, the control law is written in the following form:

$$
V(k)=\theta^{T}(k) \varphi(k)
$$

As described in [8], depending on the relative degree $r$ of the system and the nature of the transfer function of the reference model, two cases are considered:

Case i: $r=1$ and the transfer function $G_{m}\left(q^{-1}\right)$ must be strictly positive real (SPR).

Case ii: $r \succ 1$ and the transfer function $G_{m}\left(q^{-1}\right)$ must be non-strictly positive real (NSPR).

A. Synthesis of the Control Law in the Case of the Relative

Degree of the Plant $r=1$ and $G_{m}\left(q^{-1}\right)$ is Strictly

Positive Real.

In this case, we assumed that the relative degree $r$ of the plant is one and the transfer function $G_{m}\left(q^{-1}\right)$ is (SPR). So, the MRAC system can be described as shown in Fig. 3 by the block diagram below. 


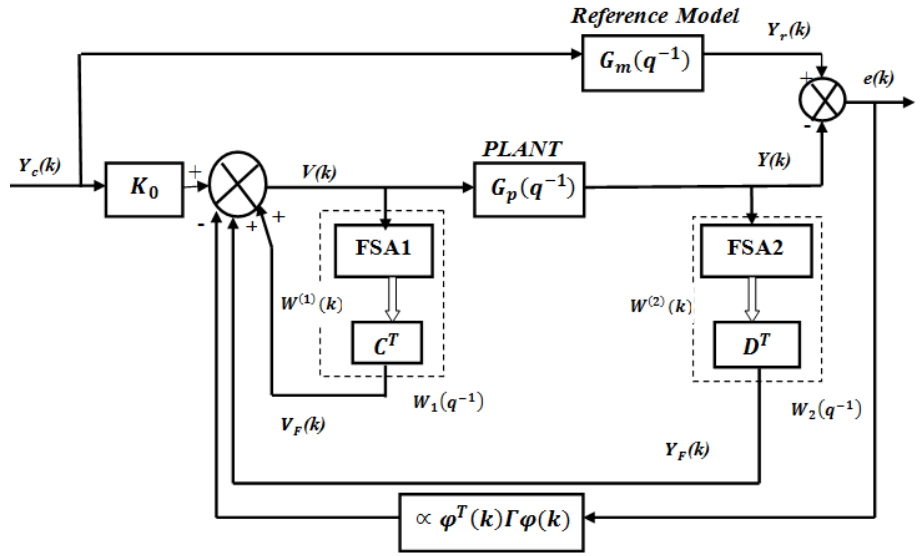

Fig. 3. A Model-Reference Adaptive Control System for Relative Degree

$$
r=1 \text {. }
$$

The controller designed by $(2 n+1)$ adjustable parameters, which represented the elements of the parameter vector $\theta(k)$ formulated by relation (10)

$$
\theta^{T}(k)=\left[\begin{array}{lll}
K_{c}(k) & C^{T}(k) & \bar{D}^{T}(k) d_{0}(k)
\end{array}\right]
$$

If a vector $\varphi(k)$ is defined as

$$
\varphi^{T}(k)=\left[Y_{c}(k) W^{(1) T}(k) W^{(2) T}(k) \quad Y(k)\right]
$$

The control law is written as:

$$
V(k)=\theta^{T}(k) \varphi(k)-\alpha \varphi^{T}(k) \Gamma \varphi(k) e(k)
$$

where $\alpha \succ 0$ and $\Gamma=\Gamma^{T}$ is a positive definite diagonal matrix

The parameters vector $\theta^{T}(k)$ can be written as follows:

$$
\theta^{T}(k)=\theta^{* T}(k)+\theta^{T}(k)
$$

Where $\theta^{* T}=\left[\begin{array}{llll}K_{0}^{*}(k) & C^{* T} \quad \bar{D}^{* T} & d_{0}^{*}(k)\end{array}\right]$ is the vector with optimal parameters, and $\theta^{T}(k)$ is the vector of errors on control parameters. Then the expression of the command law is rewritten, as follows:

$V(k)=\left(\theta^{* T}(k)+\theta^{T}(k)\right) \varphi(k)-\alpha \varphi^{T}(k) \Gamma \varphi(k) e(k)$

In this case, a constant vector $\theta^{*}$ exists such that if $\theta^{T}(k)=\theta^{* T}(k)$, then $\theta^{T}(k)=0$. So, it can be shown that the transfer function of the system will be equal to that of the reference model, and this term $\alpha \varphi^{T}(k) \Gamma \varphi(k) e(k)$ is seen to tend to zero.
Finally, in this condition the algorithm of adaptation parameters is given by the following equations:

$e(k)=Y(k)-Y_{r}(k)$

$\theta(k+1)=\theta(k)-\Gamma e(k) \varphi(k)$

$V(k)=\theta^{T}(k) \varphi(k)-\alpha \varphi^{T}(k) \Gamma \varphi(k) e(k)$

Synthesis of the Control Law in the Case of the Relative Degree $r \succ 1$ and $G_{m}\left(q^{-1}\right)$ is Non Strictly Positive Real.

In this section, we discussed the MRAC approach for the case when the relative degree $r \succ 1$ and $G_{m}\left(q^{-1}\right)$ is NSPR as described in [8], an auxiliary signal has to be fed into the reference model and the corresponding structure is described in Fig. 4.

In the condition of the relative degree $r$ is equal to one, it is easy to define a SPR reference model $G_{m}\left(q^{-1}\right)$ However, if the relative degree of the system $r \succ 1$, this assumption is not always satisfied. In this case, we assumed that there exists a urwitz polynomial $L\left(q^{(-1)}\right)$ of degree (n-1) such that $G_{m}\left(q^{-1}\right) L\left(q^{-1}\right)$ is SPR.

In this case, the error e $(\mathrm{k})$ denotes the tracking error between the output of the system Y (k) and a fictitious output $Y_{r}^{*}(k)$ which is called auxiliary error or augmented error.

$e(k)=Y(k)-Y_{r}^{*}(k)$

or

$Y_{r}^{*}(k)=Y_{r}(k)+Y_{a}(k)$

$Y_{a}(k)$ is the auxiliary output of the reference model given by the following equation:

$\mathrm{Y}_{\mathrm{a}}(\mathrm{k})=\mathrm{L}\left(\mathrm{q}^{-1}\right) \mathrm{G}_{\mathrm{m}}\left(\mathrm{q}^{-1}\right)\left(\left[\mathrm{L}^{-1}\left(\mathrm{q}^{-1}\right) \bar{\theta}^{\mathrm{T}}(\mathrm{k})-\bar{\theta}^{\mathrm{T}}(\mathrm{k}) \mathrm{L}^{-1}\left(\mathrm{q}^{-1}\right)\right] \bar{\varphi}(\mathrm{k})+\alpha \bar{\zeta}^{\mathrm{T}}(\mathrm{k}) \Gamma \bar{\zeta}(\mathrm{k}) \mathrm{e}(\mathrm{k})\right)$

where

$\bar{\xi}(k)=L^{-1}\left(q^{-1}\right) \bar{\varphi}(k)$

$\bar{\theta}^{T}(k)=\left[\begin{array}{llll}C^{T}(k) & \bar{D}^{T}(k) & d_{0}(k)\end{array}\right]$

$\bar{\varphi}^{-T}(k)=\left[W^{(1) T}(k) \quad W^{(2) T}(k) \quad Y(k)\right]$ 
Finally $[6,7,8,9,10]$, in this case the algorithm of adaptation of the parameters is given by the following equations as:

$$
\begin{aligned}
& e(k)=Y(k)-Y_{r}(k)-Y_{a}(k) \\
& \bar{\theta}(k)=\bar{\theta}(k-1)-\Gamma e(k) \bar{\xi}(k) \\
& V(k)=\bar{\theta}(k) \bar{\varphi}(k)+Y_{c}(k)
\end{aligned}
$$

\section{Simulation Results}

\section{A. Dynamic Modelling and Linearization of a Robot Manipulator}

In this section, a nonlinear six degrees of freedom robot manipulator model is employed to demonstrate the performance of the proposed MRAC approche, which is a serial open chain composed of seven rigid links connected with six rotoïde joints as discussed in our recent works [11, 12, 13]. Therefore, controlling the motion of robot is a complicated operation due to the wide number of degrees of freedom and the high nonlinearities introduce in this plant. The dynamic equations of motion for the manipulator can be expressed by the following equations:

$$
\Gamma=\mathrm{f}\left(\mathrm{q}, \dot{\mathrm{q}}, \ddot{\mathrm{q}}, \mathrm{f}_{\mathrm{e}}\right)
$$

$\Gamma_{\mathrm{i}}=\sum_{j=1}^{\mathrm{n}} \mathrm{d} \frac{\left(\frac{\partial \mathrm{L}_{\mathrm{j}}}{\partial}\right)}{\mathrm{dt}}-\frac{\partial \mathrm{L}_{\mathrm{j}}}{\partial \mathrm{q}_{\mathrm{i}}} \quad \mathrm{i}, \mathrm{j}=1, \ldots, \mathrm{n}$

where $\Gamma, \mathrm{q}, \dot{\mathrm{q}}, \ddot{\mathrm{q}}$ depicting Torques, articular positions, articular velocities and articular accelerations, $\mathrm{f}_{\mathrm{e}}$ represents the external force and $\mathrm{L}_{\mathrm{j}}$ denotes the lagrangian of the $j^{\text {th }}$ joint.

So, we have applicated the formalism of Euler-Lagrange [13], such that equation (28), we obtained this relation (29):

$$
\Gamma=\mathrm{A}(\mathrm{q}) \ddot{\mathrm{q}}+\mathrm{C}(\mathrm{q}, \dot{\mathrm{q}}) \dot{\mathrm{q}}+\mathrm{Q}(\mathrm{q})
$$

where $\mathrm{A}(\mathrm{q})$ represents the matrix of kinetic energy $(\mathrm{n} \times \mathrm{n})$; $C(q, \dot{q}) \dot{q}$ defines the vector of coriolis and centrifugal forces/torques $(\mathrm{n} \times 1) ; \mathrm{Q}(\mathrm{q})$ represents the vector of torques/forces of gravity.

Hence, the dynamic model of the above system was described by $n$ second order differential equations $[12,13]$. So, if the inertia matrix $\mathrm{A}$ is invertible for $q \in R^{n}$, we can determine the articular accelerations vector $\ddot{q}$ of each joint as relation (30).

$$
\begin{aligned}
& \ddot{\mathrm{q}}=\mathrm{f}(\mathrm{q}, \dot{\mathrm{q}}, \Gamma) \\
& \ddot{\mathrm{q}}=-\mathrm{A}(\mathrm{q})^{-1}[\mathrm{C}(\mathrm{q}, \dot{\mathrm{q}}) \dot{\mathrm{q}}+\mathrm{Q}(\mathrm{q})-\Gamma]
\end{aligned}
$$

Where $\mathrm{q}$ is the angular positions vector $(6 \mathrm{x} 1) ; \dot{q}$ is the angular velocities vector $(6 \mathrm{x} 1) ; \ddot{q}$ is the angular accelerations vector $(6 \mathrm{x} 1)$; $\Gamma$ is the input torques vector $(6 \mathrm{x} 1)$.

For the goal of linear control design, we used the input output feedback linearization approaches as $[14,15,16,17]$, to linearize the nonlinear robot dynamics model. First, we assumed that the state variables of the plant changed into state space as:

$$
\begin{gathered}
\mathrm{x}_{1}=\mathrm{q}_{1}, \mathrm{x}_{2}=\dot{\mathrm{q}}_{1}, \mathrm{x}_{3}=\mathrm{q}_{2}, \mathrm{x}_{4}=\dot{\mathrm{q}}_{2}, \mathrm{x}_{5}=\mathrm{q}_{3}, \mathrm{x}_{6}=\dot{\mathrm{q}}_{3} \\
\mathrm{x}_{7}=\mathrm{q}_{4}, \mathrm{x}_{8}=\dot{\mathrm{q}}_{4}, \mathrm{x}_{9}=\mathrm{q}_{5}, \mathrm{x}_{10}=\dot{\mathrm{q}}_{5}, \mathrm{x}_{11}=\mathrm{q}_{6}, \mathrm{x}_{12}=\dot{\mathrm{q}}_{6}
\end{gathered}
$$

According to the above, to design the affine form of model dynamic for the robot manipulator which represents multivariable and nonlinear plant, we have derived each above state variables as formulated by the system (32):

$$
\left\{\begin{array}{c}
\dot{\mathrm{X}}(\mathrm{t})=\mathrm{f}(\mathrm{X}(\mathrm{t}))+\sum_{i=1}^{\mathrm{p}} \mathrm{g}_{\mathrm{i}}(\mathrm{X}(\mathrm{t})) \mathrm{U}_{\mathrm{i}}(\mathrm{t}) \\
\mathrm{Y}_{\mathrm{i}}(\mathrm{t})=\mathrm{h}_{\mathrm{i}}(\mathrm{X}(\mathrm{t})) \\
\mathrm{i}=1,2, \ldots 6
\end{array}\right.
$$

Where $X=\left[x_{1}, x_{2} \ldots x_{n}\right]^{T} \in R^{n}$ defines the state vector; $U=\left[u_{1}, u_{2} \ldots u_{p}\right]^{T} \in R^{p}$ denotes the control input vector; $Y=\left[y_{1}, y_{2} \ldots y_{p}\right]^{T} \in R^{p}$ represents the output vector; $h_{i}(X)$ is a scalar function; $f(X)$ and $g_{i}(X)$ are n-dimensional smooth vector fields, with $i=1,2 \ldots n$.

Second, for the purpose of linearizing and decoupling the model dynamics of the system and transforming it to six linear subsystem, the feedback linearization approach as [18, 19, 20, 21] consists essentially of applied the lie derivative to each output until one or more inputs arise, as formulated in the expression (33).

$$
\text { Joints positions }\left\{\begin{array}{c}
y_{1}=h_{1}(x)=x_{1}=q_{1} \\
y_{2}=h_{2}(x)=x_{3}=q_{2} \\
y_{3}=h_{3}(x)=x_{5}=q_{3} \\
y_{4}=h_{4}(x)=x_{7}=q_{4} \\
y_{5}=h_{5}(x)=x_{9}=q_{5} \\
y_{6}=h_{6}(x)=x_{11}=q_{6}
\end{array}\right.
$$


For each joint position above, assume that the relative degree $r_{i}$ represents the smallest integer such that fully one or more of the inputs appear in the new output $y_{j}{ }^{\left(r_{j}\right)}, \mathrm{j}=1 \ldots 6$.

$$
\begin{gathered}
\mathrm{y}_{\mathrm{j}}{ }^{\left(\mathrm{r}_{\mathrm{j}}\right)}=\mathrm{L}_{\mathrm{f}}{ }^{\mathrm{r}_{\mathrm{j}}} \mathrm{h}_{\mathrm{j}}(\mathrm{x})+\sum_{\substack{i=1 \\
\mathrm{i}, \mathrm{j}=1,2, \ldots \mathrm{p}}} \mathrm{L}_{\mathrm{i}}\left(\mathrm{L}_{\mathrm{f}}{ }^{\left(\mathrm{r}_{\mathrm{j}-1}\right)} \mathrm{h}_{\mathrm{j}}(\mathrm{x})\right) \mathrm{u}_{\mathrm{i}} \\
\end{gathered}
$$

Where $L_{f}{ }^{i} h_{j}$ and $L_{g}{ }^{i} h_{j}$ are the $i^{t h}$ Lie derivatives of $\mathrm{h}_{\mathrm{j}}(\mathrm{x})$ respectively in the direction of $\mathrm{f}$ and $\mathrm{g}$.

$L_{f} h_{j}(x)=\frac{\partial h_{j}}{\partial x} f(x), L_{g} h_{j}(x)=\frac{\partial h_{j}}{\partial x} g_{i}(x)$

So, rewriting the expression (33) for each subsystem, we obtained that each one have a relative degree $r_{i}$ equal to 2 , are given by (35);

$$
\left\{\begin{array}{c}
y_{1}=h_{1}(x)=x_{1} \\
\dot{y}_{1}=L_{f} h_{1}(x)=\dot{x}_{1}=x_{2} \\
y_{1}{ }^{(2)}=L_{f}{ }^{2} h_{1}(x)+L_{g} L_{f} h_{1}(x) u \\
r_{1}=2 \\
y_{2}=h_{2}(x)=x_{3} \\
\dot{y}_{2}=L_{f} h_{2}(x)=\dot{x}_{3}=x_{4} \\
y_{2}{ }^{(2)}=L_{f}{ }^{2} h_{2}(x)+L_{g} L_{f} h_{2}(x) u \\
r_{2}=2 \\
\vdots \\
y_{6}=h_{6}(x)=x_{11} \\
\dot{y}_{6}=L_{f} h_{6}(x) \dot{x}_{11}=x_{12} \\
y_{6}{ }^{(2)}=L_{f}{ }^{2} h_{6}(x)+L_{g} L_{f} h_{6}(x) u \\
r_{6}=2
\end{array}\right.
$$

Finally, the nonlinear control law $u_{i}(t)$ applied to each joint of the robot manipulator system is formulated as the relation (36):

$$
\begin{gathered}
u_{i}(x(t))=-\frac{L_{f}^{r_{i}} h_{i}(x(t))}{L_{g}{ }^{r_{i}-1} L_{f} h_{i}(x(t))}+\frac{v_{i}(t)}{L_{g}{ }^{r_{i}-1} L_{f} h_{i}(x(t))} \\
i=1 \ldots 6
\end{gathered}
$$

By using the input-output linearizing control law given by the above relation (36), the nonlinear plant dynamic system is transformed into six decoupled and linear subsystems [22, 23, $24,25]$. Each one was discretized to facilitate the linear reference model adaptive controller design.

\section{B. Application of Control Strategy}

As the linearized plant with input output feedback linearisation is constructed, we designed a linear controller by synthesising the proposed model reference adaptive controller in the case of the relative degree $r \succ 1$ and $G_{m}\left(q^{-1}\right)$ is NSPR.

The joint1 represents a second-order and time-varying system, with relative degree $\left(r_{1}=2\right)$ as described by the following equation:

$y_{1}(k)=-a_{11}(k) y_{1}(k-1)-a_{12}(k) y_{1}(k-2)+k_{p_{1}}(k) b_{10}(k) v_{1}(k-2)$

$a_{11}(k), a_{12}(k)$ and $b_{10}(k)$ are the unknown and timevarying parameters of model 1 that is estimated with a recursive least-squares algorithm as illustrated in figure 5.

$y_{c 1}(k)$ denotes a reference input for the joint 1 , described by the following relation:

$y_{c 1}(k)=1 \forall k \geq 0$

The joint 2 represents a second-order and time-varying system, with relative degree $\left(r_{2}=2\right)$ as described by the following equation:

$$
y_{2}(k)=-a_{21}(k) y_{2}(k-1)-a_{22}(k) y_{2}(k-2)+k_{p_{2}}(k) b_{20}(k) v_{2}(k-2)
$$

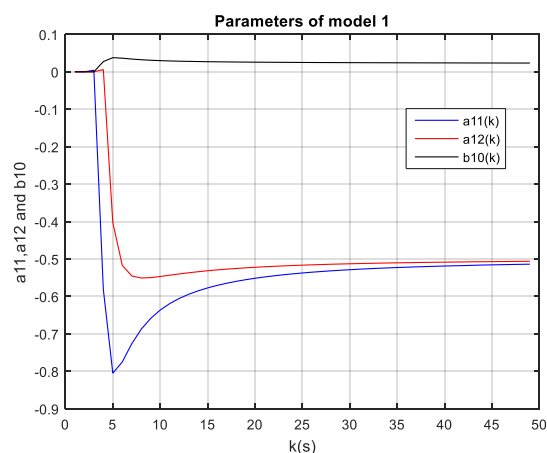

Fig. 4. The Estimated unknown and Time-Varying Parameters of Model 1.

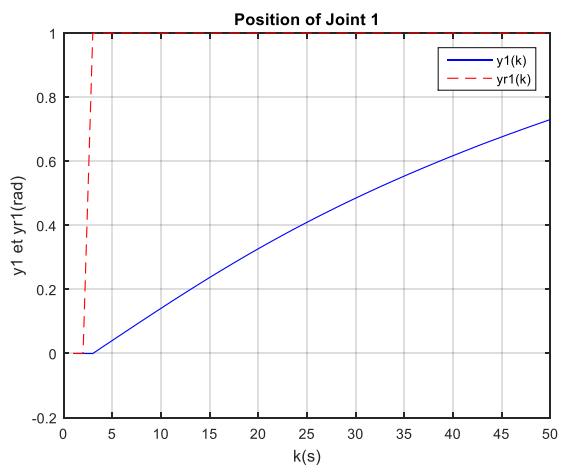

Fig. 5. The Evolutions of the Joint 1 Output $y_{1}(k)$ and Reference Model

$$
\text { Output } y_{r 1}(k) \text {. }
$$




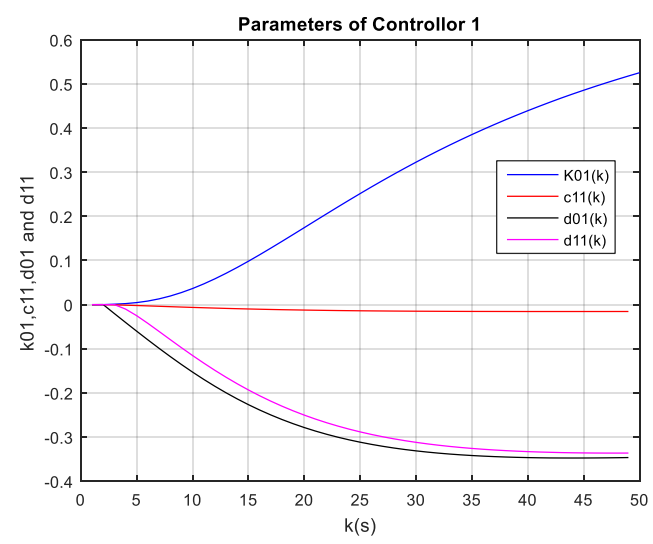

Fig. 6. Evolutions of the Controller Parameters 1.

$a_{21}(k), a_{22}(k)$ and $b_{20}(k)$ are the unknown and timevarying parameters of model 2 that is estimated with a recursive least squares algorithm as shown in Fig. 8.

$y_{c 2}(k)$ is a reference input for the joint 2 , described by the following relation:

$y_{c 2}(k)=1 \forall k \geq 0$

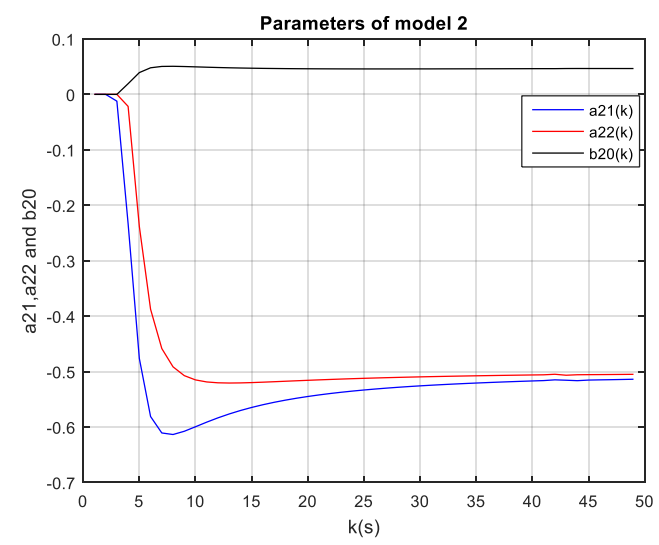

Fig. 7. The Estimated unknown and Time-Varying Parameters of Model 2.

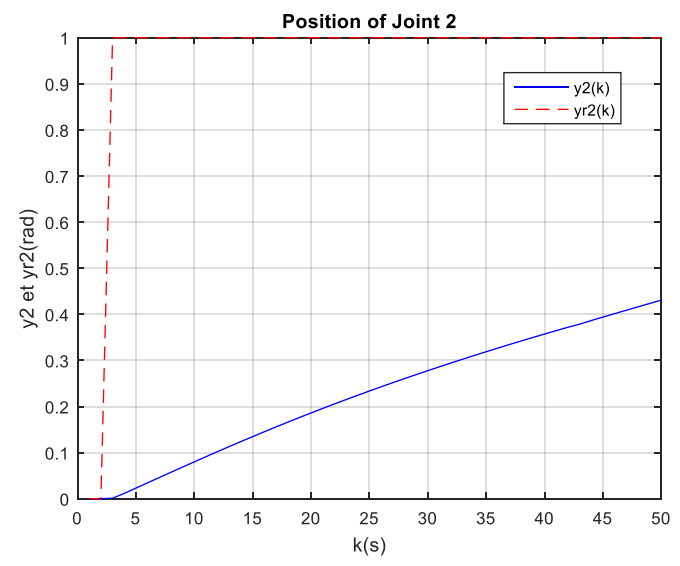

Fig. 8. The Evolutions of the Joint1Output $y 2(k)$ and Reference Model

$$
\text { Output } y_{r 2}(k) \text {. }
$$

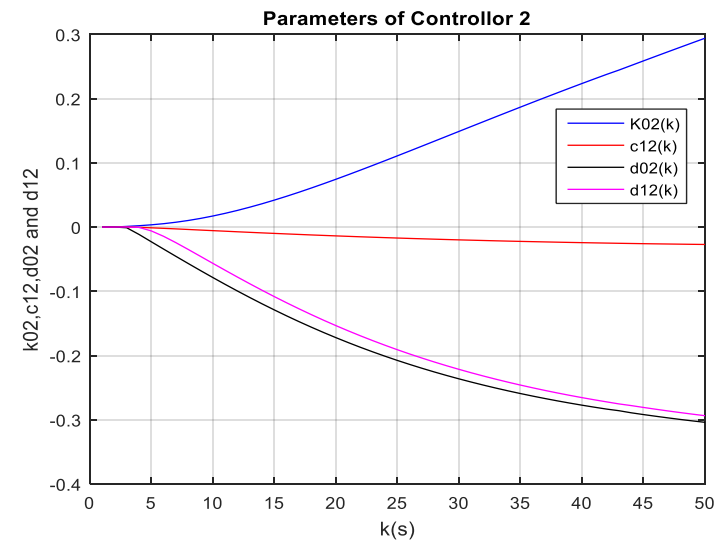

Fig. 9. The Adjustment Parameters of the Controller 2.

The joint 3 represents a second-order and time-varying system, with relative degree $(r 3=2)$ as determined by the following equation:

$y_{3}(k)=-a_{31}(k) y_{3}(k-1)-a_{32}(k) y_{3}(k-2)+k_{p_{3}}(k) b_{30}(k) v_{3}(k-2)$

$a_{31}(k), a_{32}(k)$ and $b_{30}(k)$ are the unknown and timevarying parameters of model 3 that is estimated with a recursive least-squares algorithm as shown in Fig. 11.

$y_{c 3}(k)$ is a reference input for the joint 3 , is given by the following relation:

$y_{c} 3(k)=1 \forall k \geq 0$

The joint 4 represents a second-order and time-varying system, with relative degree $(r 4=2)$ as formulated by the following equation (43):

$y_{4}(k)=-a_{41}(k) y_{4}(k-1)-a_{42}(k) y_{4}(k-2)+k_{p_{4}}(k) b_{40}(k) v_{4}(k-2)$

$a_{41}(k), a_{42}(k)$ and $b_{40}(k)$ are the unknown and timevarying parameters of model 4 that is estimated with a recursive least squares algorithm as demonstrated in Fig. 14.

$y_{c} 4(k)$ is a reference input for the joint 4 , was elaborated by the following relation (44):

$y_{c 4}(k)=1 \forall k \geq 0$

The joint 5 represents a second-order and time-varying system, with relative degree $(r 5=2)$ as given by the following equation (45):

$y_{5}(k)=-a_{51}(k) y_{5}(k-1)-a_{52}(k) y_{5}(k-2)+k_{p_{5}}(k) b_{50}(k) v_{5}(k-2)$

$a_{51}(k), a_{52}(k)$ and $b_{50}(k)$ are the unknown and timevarying parameters of model 5 that is estimated with a recursive least squares algorithm as shown in Fig. 17.

$y_{c} 5(k)$ is a reference input for the joint 5 , represented by the following relation (46): 
$y_{c} 5(k)=1 \forall k \geq 0$

The joint 6 represents a second-order and time-varying system, with relative degree $\left(r_{6}=2\right)$ as determined by the following equation (47):

$y_{6}(k)=-a_{61}(k) y_{6}(k-1)-a_{62}(k) y_{6}(k-2)+k_{p_{6}}(k) b_{60}(k) v_{6}(k-2)$

$a_{61}(k), a_{62}(k)$ and $b_{60}(k)$ are the unknown and timevarying parameters of model 6 that is estimated with a recursive least-squares algorithm as shown in Fig. 20.

$y_{c} 6(k)$ is a reference input for the joint 6 , denoted by the following relation (48):

$y_{c} 6(k)=1 \forall k \geq 0$

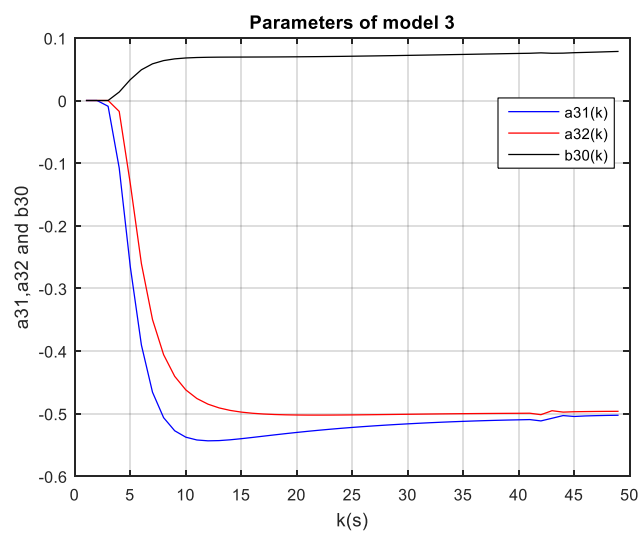

Fig. 10. The Estimated unknown and Time-Varying Parameters of Model 3.

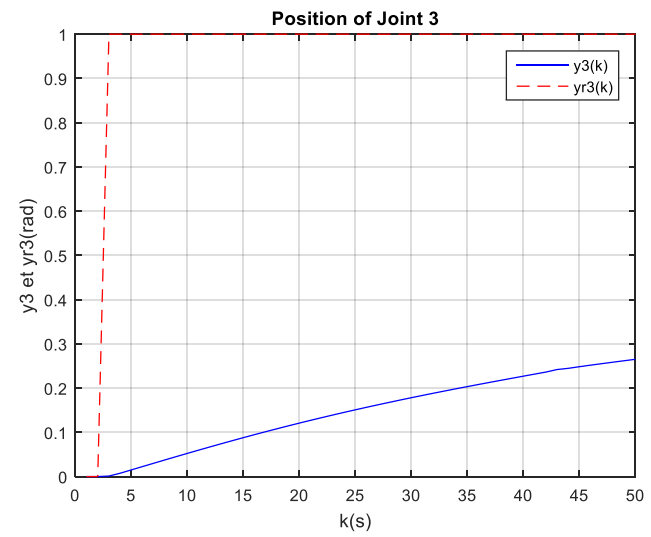

Fig. 11. The Evolutions of the Joint1Output $y 3(k)$ and Reference Model

$$
\text { Output } y_{r 3}(k) \text {. }
$$

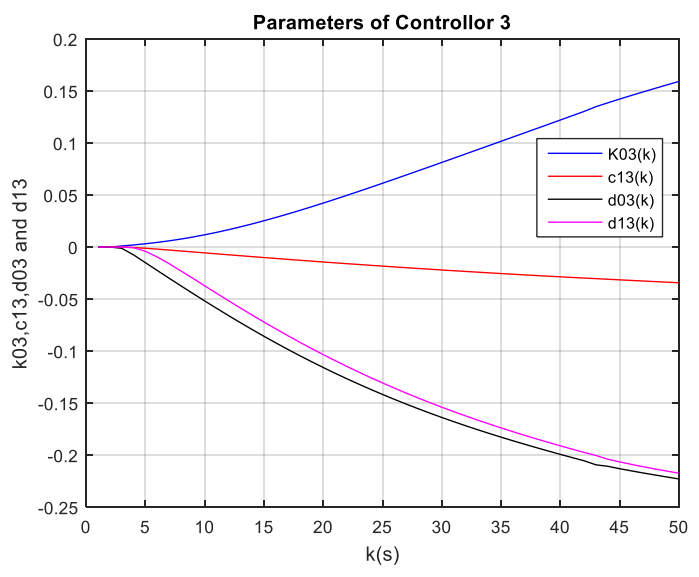

Fig. 12. The Adjustment Parameters of the Controller 3.

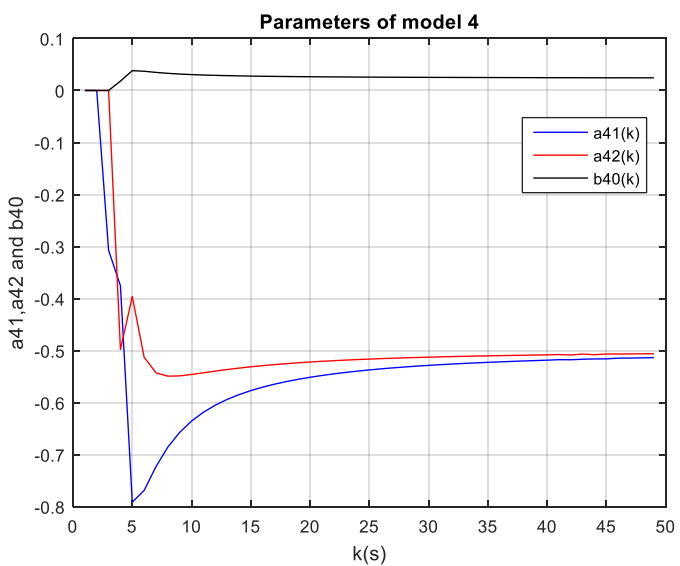

Fig. 13. The Estimated unknown and Time-Varying Parameters of Model 4.

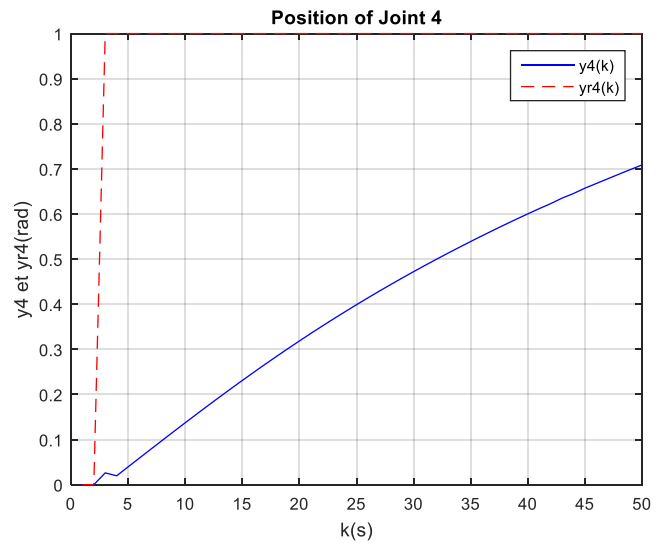

Fig. 14. The Evolutions of the Joint1Output $y 4(k)$ and Reference Model Output $y_{r 4}(k)$. 


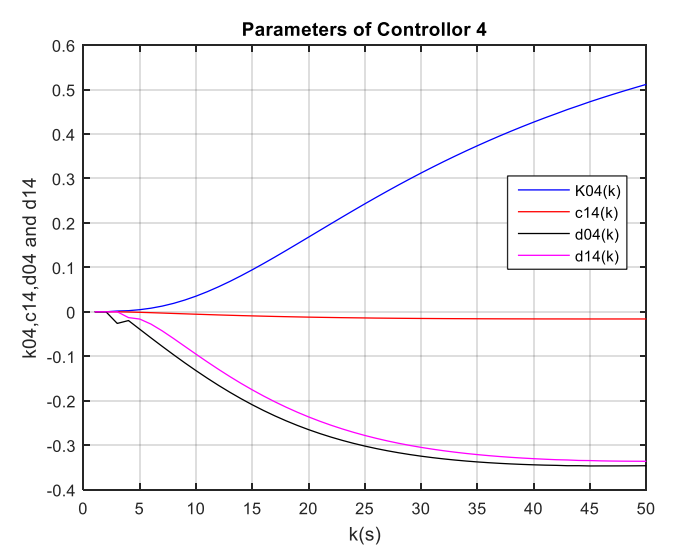

Fig. 15. The Adjustment Parameters of the Controller 4.

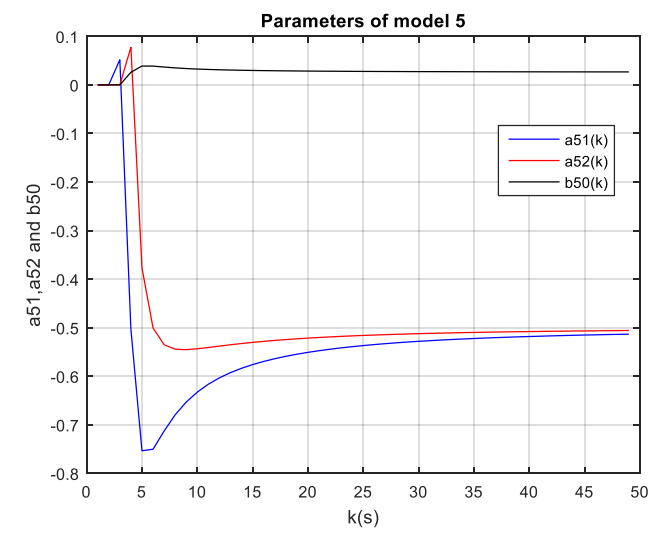

Fig. 16. The Estimated unknown and Time-Varying Parameters of Model 5.

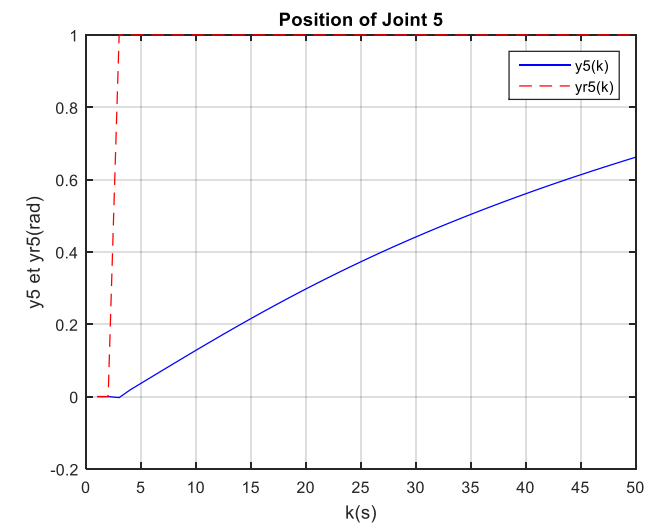

Fig. 17. The Evolutions of the Joint1Output $y 5(k)$ and Reference Model Output $y_{r 5}(k)$.

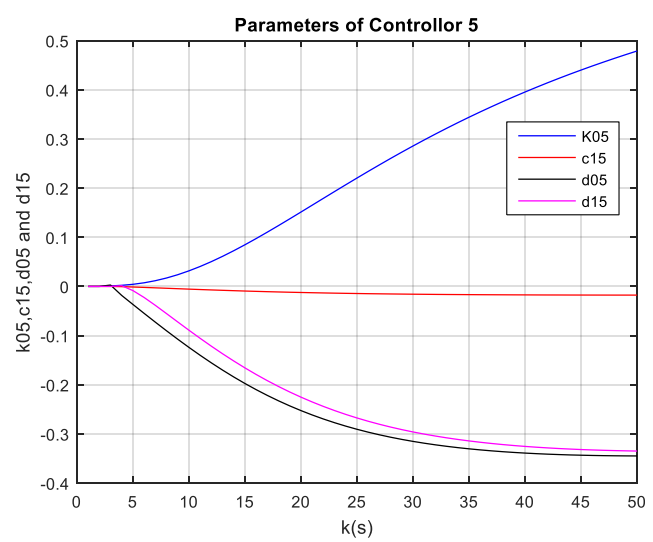

Fig. 18. The Adjustment Parameters of the Controller 5.

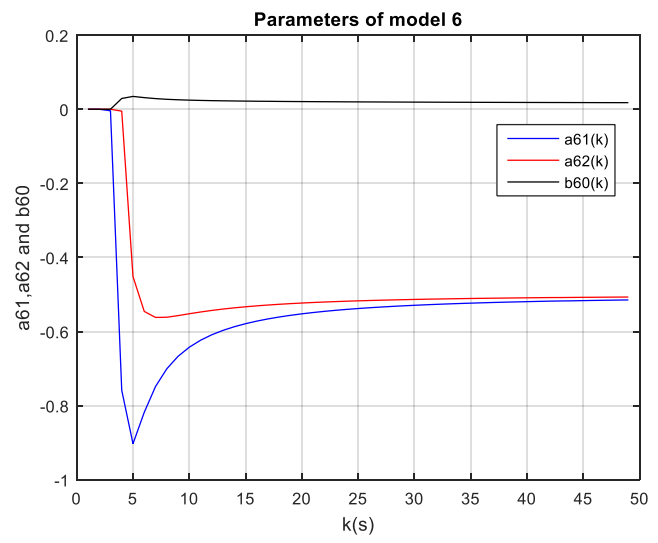

Fig. 19. The Estimated unknown and Time-Varying Parameters of Model 6.

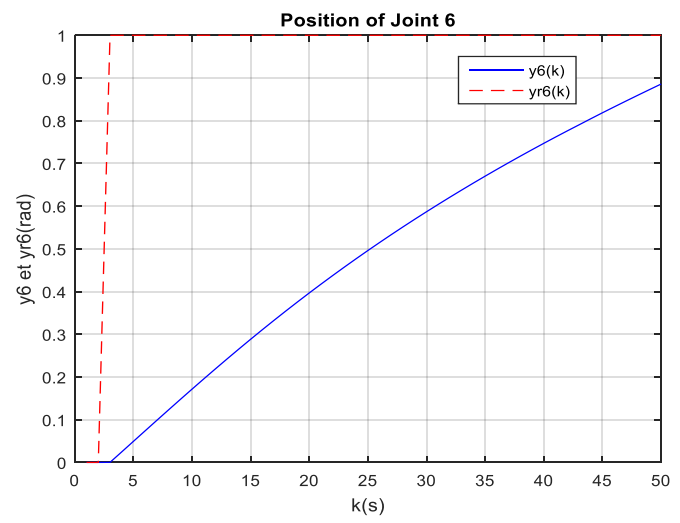

Fig. 20. The Evolutions of the Joint1Output $y_{6}(k)$ and Reference Model Output $y r 6(k)$. 


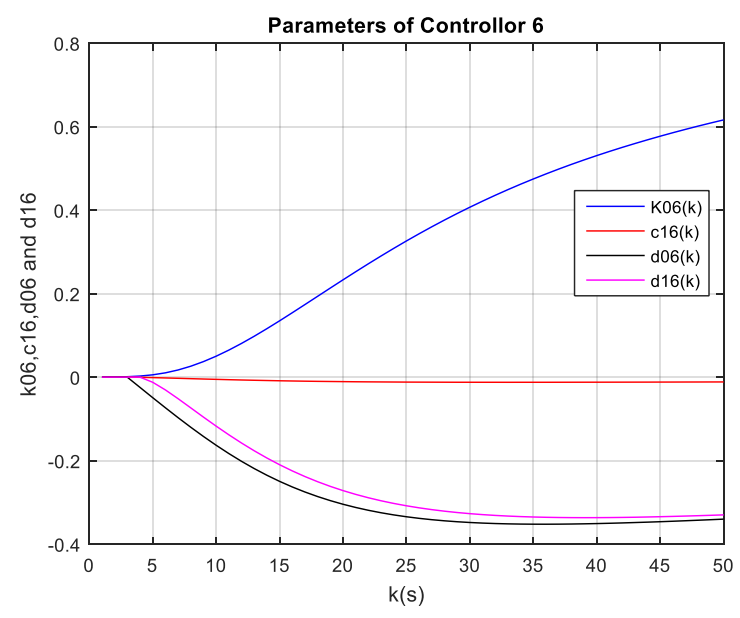

Fig. 21. The adjustment parameters of the controller 6 .

Through the simulation results, as illustrated in figures $6,9,12,15,18,21$, each one represents the evolution of the joint output and reference model output, one notes that each joint output converge to the reference model output, so each adaptive controller designed by parameters illustrated respectively in figures $7,10,13,16,19,22$ was demonstrated a satisfactory tracking performance.

\section{CONCLUSION}

In this paper, a general class of discrete time adaptive control algorithms has developed and has illustrated that, under suitable cases, they will be convergent. This algorithm is used for SISO and MIMO plants. Two fundamental cases of controller design techniques are discussed in detail when the relative degree of the system equal to one and the transfer function of the reference model is assumed to be strictly positive. Also, the condition denotes that the relative degree greater than one and the transfer function supposed to be nonstrictly positive real was called an augmented control architecture.

The contribution of this paper consists of motivated the studied analysis through its appliance to a robot manipulator with six degrees of freedom that is represents nonlinear, dynamic, multivariable and decoupled system. After linearization using the input-output feedback linearization and decoupling method, the nonlinear MIMO system was transformed into six independent SISO linear subsystems each one was represented by a relative degree equal to two with unknown and time-varying parameters. So, each linear subsystem has discretized to facilitate the linear MRAC design. However, the unknown and time-varying parameters of each model are estimated with a recursive least-squares algorithm. Finally, the control taw of the augmented MRAC has been successfully implemented to each model as shown in the above simulation results.

As a perspective of our work, we will extend these researches for the plants with disturbances.

\section{REFERENCES}

[1] K.J. Aström et B. Wittenmark, "Adaptive control”, Addisson-Wesley, 1989.

[2] MW. Spong, S.Hutchinson, and M. Vidyasagar. "Robot Dynamics and Control , "Second Edition. January 28, 2004.

[3] I. D. Landau, R. Lozano, M. M'Saad and A. Karimi. "Adaptive Control Algorithms, Analysis and Applications, "Second Edition. (C) SpringerVerlag London Limited 2011.

[4] I. D. Landau, R. Lozano, M. M'Saad. "Adaptive Control, " Springer Verlag, 1997.

[5] I.D.Landau,“ From robust control to adaptive control, " Control Eng. Practice, vol.7, N10, pp.1113-1124, April 2002.

[6] K. S. Narendra, L. S. Valvani. "Stable discrete adaptive control, " IEEE Transactions on Automatic Control, vol. 25, 1980.

[7] K. S. Narendra, Y. H. Lin. "A new error model for adaptive sytems," IEEE Transactions on Automatic Control, vol. 25(3), 1980.

[8] K.S.Narendra and A.M. Annaswamy. "Stable Adaptive Systems, " Englewood Cliffs, NJ: Prentice Hall. 1989

[9] S.S. Sastry et M. Bodson, "Adaptive Control: Stability, Convergence, and Robustness, " Prentice Hall, Englewood Cliffs, NJ, 1989.

[10] H. Kaufman, I. Barkana, and K. Sobel. "Direct adaptive control algorithms, " Springer, New York, 1994

[11] W.Ghozlane and J.Knani."Modelling and Simulation Using Mathematical and CAD Model of a Robot with Six Degrees of Freedom," CEIT-2016, $4^{\text {th }}$ International Conference on Control Engineering \& Information Technology.16-18 December 2016Hammamet, Tunisia. ISBN: 978-1-5090-1055-4 @ 2016.On IEEE.

[12] W.Ghozlane and J.Knani."Nonlinear Control of MIMO System Using Feedback Linearization Control Method and PD Controller for Tracking Purpose, " CEIT-2017, 5 International Conference on Control Engineering \& Information Technology on IEEE.17-19 December 2017Sousse, Tunisia.

[13] W.Ghozlane and J.Knani. "Non Linear Control via Input-Output Feedback Linearisation of a Robot Manipulator, "Advances in Science,Technology and Engineering Systems Journal Vol.3,No,5,374381.2018.

[14] L. Sciavicco and B. Siciliano, "Modeling and control of robot manipulators," 2nd edition, Springer-Verlag London Limited, 2000.

[15] A. Isidori. "Nonlinear Control Systems," Springer Verlag, 1989.

[16] W. Li J. J. E. Slotine. “Applied Nonlinear Control, ” Prentice Hall, 1991.

[17] H. Khalil. "Nonlinear Systems, " Prentice Hall, Upper Saddle River, Second Edition, 1996

[18] Nelles. O, "Nonlinear system identification, ” Springer, 2001.

[19] A. Astolfi, D. Karagiannis, and R. Ortega. "Nonlinear and adaptive control with applications, Communications and Control Engineering," Springer-Verlag, 2007.

[20] J.P. Corriou. "Process Control Theory and Applications, " SpringerVerlag London 2004.

[21] S. Skogestad and I. Postlethwaite. "Multivariable Feedback Control. Analysis and Design," Wiley, Chichester. New York . Brisbane. Toronto . Singapore, Second Edition . 2001.

[22] Ortega and M.W. Spong. "Adaptive motion control of rigid robots, " In Proc. IEEE 27th Conf. Decision and Control, pp. 1575-1584. 1988 (Austin, TX).

[23] J.E.Slotine and $\mathrm{W}$. $\mathrm{Li}$, "On the adaptive control of robot manipulators, " Int. J. Robot. Res.6:49-59. 1987.

[24] W. Li, and J.J.E. Slotine, . "An indirect adaptive controller," Sys. Control Lett. 12:259-266. 1989.

[25] J.J. Craig, P.Hsu, and S.S. Sastry. "Adaptive control of mechanical manipulators, ” Int. J. Robot. Res. 6:16-28. 1987. 